\title{
THE SMITH INVARIANTS OF A MATRIX SUM
}

\author{
ROBERT C. THOMPSON ${ }^{1}$
}

\begin{abstract}
A divisibility relation is proved relating the invariant factors of a sum of integral matrices.
\end{abstract}

Let $R$ be a principal ideal domain, that is, a commutative ring without zero divisors in which every ideal is principal. Let $A$ be a matrix over $R$. It is well known [1] that for the relation "equivalence of matrices," namely $A \rightarrow U A V$ where $U, V$ are invertible matrices over $R$, there exists a complete set of invariants, the invariant factors of $A$. If $A$ is $n \times n$, its invariant factors are elements $h_{1}(A), \ldots, h_{n}(A)$ of $R$ satisfying the divisibility conditions $h_{1}(A)|\cdots| h_{n}(A)$. Very much, in fact, almost full information is now available [2] about the restrictions imposed upon the invariant factors of matrices $A, B, C$ when it is required that $C=A B$. Nothing, however, seems to be known about the behavior of invariant factors when matrices are added, and the objective of this paper is to obtain a result along these lines.

Theorem 1. Let $A, B, C=A+B$, be matrices over $R$, with invariant factors

$$
h_{1}(A)|\cdots| h_{n}(A), \quad h_{1}(B)|\cdots| h_{n}(B), \quad h_{1}(C)|\cdots| h_{n}(C),
$$

respectively. Let the invariant factors of

$$
D=\operatorname{diag}\left(h_{1}(A), \ldots, h_{n}(A), h_{1}(B), \ldots, h_{n}(B)\right)
$$

be $h_{1}(D)|\cdots| h_{2 n}(D)$. Then

$$
h_{i}(D) \mid h_{i}(C), \quad i=1, \ldots, n .
$$

Proof. Let $D_{A}=\operatorname{diag}\left(h_{1}(A), \ldots, h_{n}(A)\right)$. Unimodular matrices $U_{1}, U_{2}, V_{1}, V_{2}$ over $R$ exist such that

$$
A=U_{1} D_{A} V_{1}, \quad B=U_{2} D_{B} V_{2}
$$

Then the matrix

$$
M=\left[\begin{array}{cc}
U_{1} & U_{2} \\
0 & I
\end{array}\right]\left[\begin{array}{cc}
D_{A} & 0 \\
0 & D_{B}
\end{array}\right]\left[\begin{array}{cc}
V_{1} & 0 \\
V_{2} & I
\end{array}\right]=\left[\begin{array}{cc}
C & \cdot \\
\cdot & \cdot
\end{array}\right]
$$

is $2 n \times 2 n$, has the same invariant factors as $D$ and has $C$ as an $n \times n$ submatrix. Now, a recent result of the author [3] asserts that invariant factors $h_{1}(M)\left|h_{2}(M)\right| \cdots$ of a matrix $M$ and the invariant factors of a submatrix $C$

Received by the editors November 28, 1977.

AMS (MOS) subject classifications (1970). Primary 15A36.

${ }^{1}$ The preparation of this paper was supported in part by Grant 77-3316, United States Air Force. 
satisfy

$$
h_{i}(M) \mid h_{i}(C), \quad i=1,2, \ldots
$$

Since here $h_{i}(M)=h_{i}(D)$, the proof is complete.

In fact, further information about invariant factors of submatrices is given in [3]. However, this additional information yields nothing in the present case, although it will yield additional information about the invariant factors of $A, B, A+B$, when $A$ and $B$ are rectangular matrices.

As an application of Theorem 1, we prove an explicit inequality relating the invariant factors of $A, B, A+B$.

Theorem 2. Let the invariant factors of $A, B, C=A+B$, be as defined in the statement of Theorem 1. Then

$$
\operatorname{gcd}\left(h_{i}(A), h_{j}(B)\right) \mid h_{i+j-1}(A+B)
$$

for any indices $i, j$ with $1 \leqslant i, j \leqslant n, i+j-1 \leqslant n$, where gcd denotes greatest common divisor.

Proof. It suffices to prove that $\operatorname{gcd}\left(h_{i}(A), h_{j}(B)\right) \mid h_{i+j-1}(D)$, and for this we may focus attention upon elementary divisors for one prime $p$, i.e., upon one prime factor $p$ of $h_{n}(A) h_{n}(B)$. Let

$$
p^{e_{i}}\left\|h_{i}(A), p^{f_{i}}\right\| h_{i}(B), p^{g_{i}} \| h_{i}(D)
$$

be the exact powers of $p$ appearing in $h_{i}(A), h_{i}(B), h_{i}(D)$, respectively; $e_{1} \leqslant \cdots \leqslant$ $e_{n}, f_{1} \leqslant \cdots \leqslant f_{n}, g_{1} \leqslant \cdots \leqslant g_{2 n}$. The power on $p$ in the $t$ th determinantal divisor of $D$ is then

$$
g_{1}+\cdots+g_{t}=\min _{\substack{r, s \\ r+s=t}}\left(e_{1}+\cdots+e_{r}+f_{1}+\cdots+f_{s}\right) .
$$

We wish to prove that $\min \left(e_{i}, f_{j}\right) \leqslant g_{i+j-1}$. From (1) we certainly have

$$
g_{1}+\cdots+g_{i+j-1}=e_{1}+\cdots+e_{r}+f_{1}+\cdots+f_{s}
$$

for certain $r, s$ with $r+s=i+j-1$. We cannot have both $r<i$ and $s<j$, so suppose $s \geqslant j$. Using (1) again, we have

$$
g_{1}+\cdots+g_{i+j-2} \leqslant e_{1}+\cdots+e_{r}+f_{1}+\cdots+f_{s-1} .
$$

Combining (2) and (3), we obtain $g_{i+j-1} \geqslant f_{s} \geqslant f_{j} \geqslant \min \left(e_{i}, f_{j}\right)$. The proof is complete.

In many instances, Theorem 2 gives no information since $h_{i}(A), h_{j}(B)$ are often relatively prime. This, however, is not a failure of Theorem 2 because there are many instances in which the invariant factors of $A+B$ can be quite arbitrary even when those of $A$ and $B$ are fixed. For example, if $x \mid y$ and

$$
A=\left[\begin{array}{ll}
1 & x \\
0 & 1
\end{array}\right], \quad B=\left[\begin{array}{cc}
-1 & 0 \\
y & -1
\end{array}\right],
$$

then $h_{1}(A)=h_{2}(A)=1, h_{1}(B)=h_{2}(B)=1, h_{1}(C)=x, h_{2}(C)=y$, with $x, y$ quite arbitrary. However, Theorem 2 does cover nontrivially the following sort of situation: If prime $p \mid h_{1}(A)$ and $p \mid h_{1}(B)$ then $p$ must divide each element of $A$ and $B$, therefore also each element of $C$, and so $p$ necessarily is a divisor of $h_{1}(C)$. 
The subscript $i+j-1$ is the smallest for which Theorem 2 is true. Indeed, take $R=Z$, and set

$$
\begin{gathered}
A=I_{i-1} \dot{+}(2) \dot{+} 2 I_{j-1}+2 I_{n-i-j+1}, \\
B=-2 I_{i-1} \dot{+}(2) \dot{+}-I_{j-1}+2 I_{n-1-j+1}
\end{gathered}
$$

where $I$ denotes an identity matrix and $\dot{+}$ direct sum. Then $h_{i}(A)=2, h_{j}(B)=2$, but $h_{i+j-2}(C)=1$.

\section{REFERENCES}

1. M. Newman, Integral matrices, Academic Press, New York, 1972.

2. R. C. Thompson, Smith invariants of products of integral matrices (in preparation).

3. __ Interlacing inequalities for invariant factors, Linear Algebra and Appl. 24 (1979), 1-31.

Institute for Algebra, Department of Mathematics, University of California at Santa Barbara, SANta Barbara, California 93106 